\title{
Isolation and Functional Analysis of the Promoter Sequence of the Cry $j$ 1 Gene, Which Encodes a Major Allergenic Protein in the Pollen of Japanese Cedar (Cryptomeria japonica)
}

\author{
Shinya YAMADA, Shinya KAJITA*, Takahiro SHIOKAWA and Noriyuki MOROHOSHI \\ Graduate School of Bio-Applications and Systems Engineering, Tokyo University of Agriculture and \\ Technology, Koganei, Tokyo 184-8588, Japan \\ *Corresponding author E-mail address: kajita@cc.tuat.ac.jp \\ Received 26 August 2002; accepted 10 February 2003
}

\begin{abstract}
We isolated promoter sequences of the Cry $j 1$ gene-which encodes an allergenic protein in the pollen of Japanese cedar-by an inverse-PCR technique using genomic DNA as the template. One of the isolated promoter sequences conferred pollen-associated expression on a fusion construct that included a gene for $\beta$-glucuronidase (GUS) in transgenic tobacco plants. Histochemical analysis of GUS expression using four deletion mutants of this promoter sequence revealed that expression differed among pollen grains at different stages of maturation and that a 223-bp 5'- untranslated region of the gene was sufficient for spatial and temporal expression of the gene in pollen and pollen tubes. Our results indicate that the promoter sequence might be adequate for active pollen-associated expression in tobacco, as it is in Cryptomeria japonica.
\end{abstract}

Key words: allergenic proteins, Cry 11 gene, Cryptomeria japonica, transgenic tobacco.

Japanese cedar, Sugi (Cryptomeria japonica D. Don), is one of the most important conifers to commercial lumber production in Japan. However, two major allergenic proteins, encoded by the $C r y j$ $I$ and $C r y j 2$ genes, are associated with the pollen derived from male flowers, and they cause serious pollinosis which has become a significant medical problem in Japan (Yasueda et al., 1983; Taniai et al., 1988; Sakaguchi et al., 1990). The symptoms caused by these proteins have been investigated in depth, and the mechanisms of pollinosis and methods for prevention and treatment have also been reported (Horiguchi and Saito, 1964; Shibamura and Nishimura, 1991).

Yasueda et al. (1983) succeeded in purifing Cry $j$ 1 protein from Sugi pollen and showed that it is a basic glycoprotein of approximately $40 \mathrm{kDa}$ with a pI of 8.9-9.2. Knowledge of the amino acid sequence of the purified Cry $\mathrm{j} 1$ protein has allowed the isolation of two cDNA clones by reverse transcription and the polymerase chain reaction (Sone $e t$ al., 1994). Analysis of amino acid sequences deduced from the CDNAs and enzymatic analysis of recombinant Cry $\mathrm{j} 1$ protein revealed that $\mathrm{Cry} j \mathrm{l}$ belongs to the pectate lyase family (Sone et al, 1994; Taniguchi et al., 1995). Furthermore, local- ization of the transcript and the protein product of the Cry $j 1$ gene has been investigated, with Wang et al. (1998) reporting that $C r y j 1$ transcripts were abundant in pollen but not detectable in other tissues such as leaves, stems, roots, and female strobili. Fukui et al. (2001) and Futamura et al. (2002) also detected Cry $j 1$ transeripts in male strobili. Furthermore, it was reported that the Cry $j 1$ protein was localized on sexines, nexines, orbicles, and the cell walls of developing cells in pollen (MikiHirosige, 1994). In addition to expression of the Cry $j 1$ gene, the specific or preferential expression of genes for pectate lyase-like proteins in pollen has been reported in several other plant species (Wing et al., 1989; Budelier et al., 1990; McCormick, 1991; Rafner et al., 1991; Rogers et al., 1992; Turcich et al., 1993; Kim et al., 1994). Genes for polygalacturonase-like proteins, such as Cry $j 2$, and the gene for a pectin methyl esterase, with pectin-metabolizing enzymes, are also expressed in pollen (Brown and Crouch, 1990; Niogret et al., 1991; Mu et al., 1994; Wakeley et al., 1998). These pectin-metabolizing proteins, including the products of the Cry $j 1$ and Cry $j 2$ genes, probably play important roles in the development of pollen and the growth of pollen tubes, for which the 
rapid turnover of pectin is required (McCormick, 1991). However, to our knowledge, the promoter sequence of the Cry $j l$ gene and its activity in transgenic plants have not been reported.

In this report, we describe the isolation of the promoter sequence of the Cry $j 1$ gene by inversePCR (IPCR) using the genomic DNA of $C$. japonica as the template. To clarify the role of this sequence, we produced transgenic tobacco plants that harbored a construct in which the promoter sequence, or a variant of this sequence with a specific deletion, was fused with a gene for $\beta$-glucuronidase (GUS).

To obtain a DNA fragment that corresponded to the Cry $j 1$ promoter, we performed IPCR using DNA from needles of $C$. japonica (clone Ibaraki no. 1). Total DNA $(5 \mu \mathrm{g})$ was digested with BglII for 6 $h$ at $37^{\circ} \mathrm{C}$, and then fragments were self-ligated with T4 DNA ligase (Ligation Kit ver. 2; Takara Shuzo, Kyoto, Japan). To amplify the promoter sequence of Cry $j$ 1, we synthesized two sets of primers whose sequences were derived from the sequence of Cry $j 1 \mathrm{cDNA}$ and used the self-ligated total DNA as a template for IPCR. The nucleotide sequences of the primers were follows: $\mathrm{F} 1,5^{\prime}$ TGGGCACAAAACAGAATGAAGCTCGC-3'; R1, 5' - TTGGTAGCAG AGTAGAATGTC- 3'; F2, 5'-CAGATTGTGCAGTGGGCTTCGGA-3'; and R2, 5'-ATACGGCTATGCTATGATTATGA-3'. The first IPCR was performed with the self-ligated total DNA as template, primers (F1 and R1), and Taq DNA polymerase (Ex Taq; Takara Shuzo). One -tenth of the reaction mixture after the first IPCR was used as the template for a second IPCR with primers $\mathrm{F} 2$ and $\mathrm{R} 2$. The conditions for both the first and second IPCRs were as follows: incubation for 5 min at $94{ }^{\circ} \mathrm{C} ; 30$ cycles of $1 \mathrm{~min}$ at $94^{\circ} \mathrm{C}, 1 \mathrm{~min}$ at 56 ${ }^{\circ} \mathrm{C}$, and $5 \mathrm{~min}$ at $72^{\circ} \mathrm{C}$; and a final incubation for 5 min at $72^{\circ} \mathrm{C}$. Amplified DNA fragments were cloned into the pT7Blue vector (Novagen, Madison, WI) and partial sequences were determined. The amplified DNA fragment (approximately $1.8 \mathrm{kbp}$ ) obtained after the second IPCR included sequences derived from both the open reading frame and the promoter of the Cry $j 1$ gene. Therefore, to obtain the fragment that was derived only from the 5'untranslated region and which contained the promoter sequence of the gene, we performed PCR with a new set of primers, using genomic DNA as the template. The nucleotide sequences of these primers were as follows: F3, 5'-GATCTAAACTTTAAATGTGAAAAATGATCT-3'; and R3, 5'-TTTTTGGTAGCAGAGTGTAGAATTTCTTTC- 3 '.

From this procedure we obtained a DNA fragment of $1,511 \mathrm{bp}$ that corresponded to the promoter sequence. We determined the entire sequences of nine independent amplified promoter fragments of 1,511 bp after cloning them into the pT7Blue vector. Analyses of these sequences revealed that the nine promoter sequences could be divided into two groups with differences at seven positions (accession numbers AB080241 and AB080242). In a previous study, Wang et al. (1998) demonstrated that $C$. japonica has five to eight copies of the Cry $j$ 1 gene per diploid genome. In addition, four distinct forms of the Cry $\mathrm{j} 1$ protein were identified by Yasueda et al. (1983). These observations are consistent with our present findings, and it appears that there are at least two different Cry $j 1$ genes that encode a pectate lyase in the genome of $C$. japonica.

Comparison of promoter sequences between the Cry $j 1$ and Cry $j 2$ genes (accession numbers $\mathrm{AB} 084068$ and $\mathrm{AB} 084069$ ) revealed that both genes have the same 11-bp cis element TATAGAAAGAA located at positions -34 to $-24($ Cry $j$ 1) and positions -26 to $-16(\mathrm{Cry} j 2)$ relative to the codon for the initiation of translation. In addition, the sequence of the isolated promoter of the Cry $j I$ gene revealed a putative pollen-related cis-regulatory sequence (GAACATGTGT), which resembled the $56 / 59$ box (GAAMTTGTGA, where M

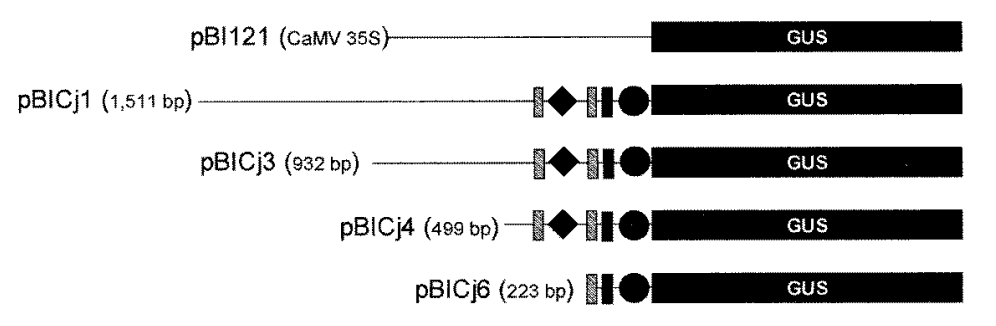

\begin{tabular}{|ll}
\hline 56/59-like box & Putative TATA box \\
Putative LIM-binding sequence & Cryj $1 /$ CYy $/ 2$ consensus sequence \\
\hline
\end{tabular}

Fig. 1 Cry $j 1$ promoter-GUS constructs used for transformation of tobacco plants. The 5 untranslated region of the Cry $j 1$ promoter and the GUS - coding sequence are indicated as a line and a bar, respectively. Portions of putative cis elements are also indicated. 

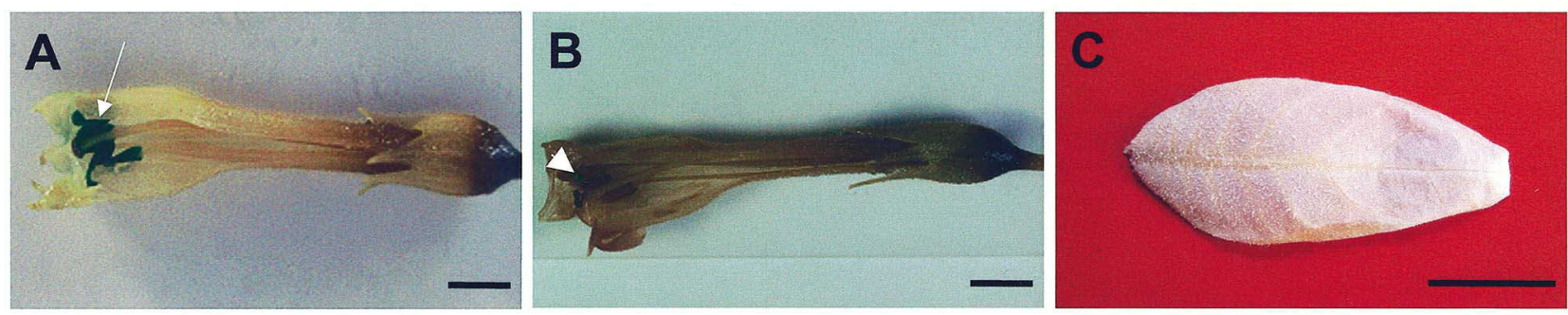

Fig. 2 Histochemical GUS assays in flowers of transgenic plants transformed with pBICj 1 before (A) and after (B) pollen dispersion. The assay was also performed with transgenic leaf tissue $(C)$. The tissues were stained with 5-bromo-4-chloro-3 indolyl- $\beta$-D-glucuronide (X-Gluc) and then decolorized (extraction of chlorophyll) by ethanol. Stamens before pollen dispersion (A, arrow) were stained blue by the procedure. In contrast, no positive signals were observed in stamens after pollen dispersion (B). The faint blue staining observed in B is due to residual pollen (arrowhead). Bars $=1 \mathrm{~cm}$.

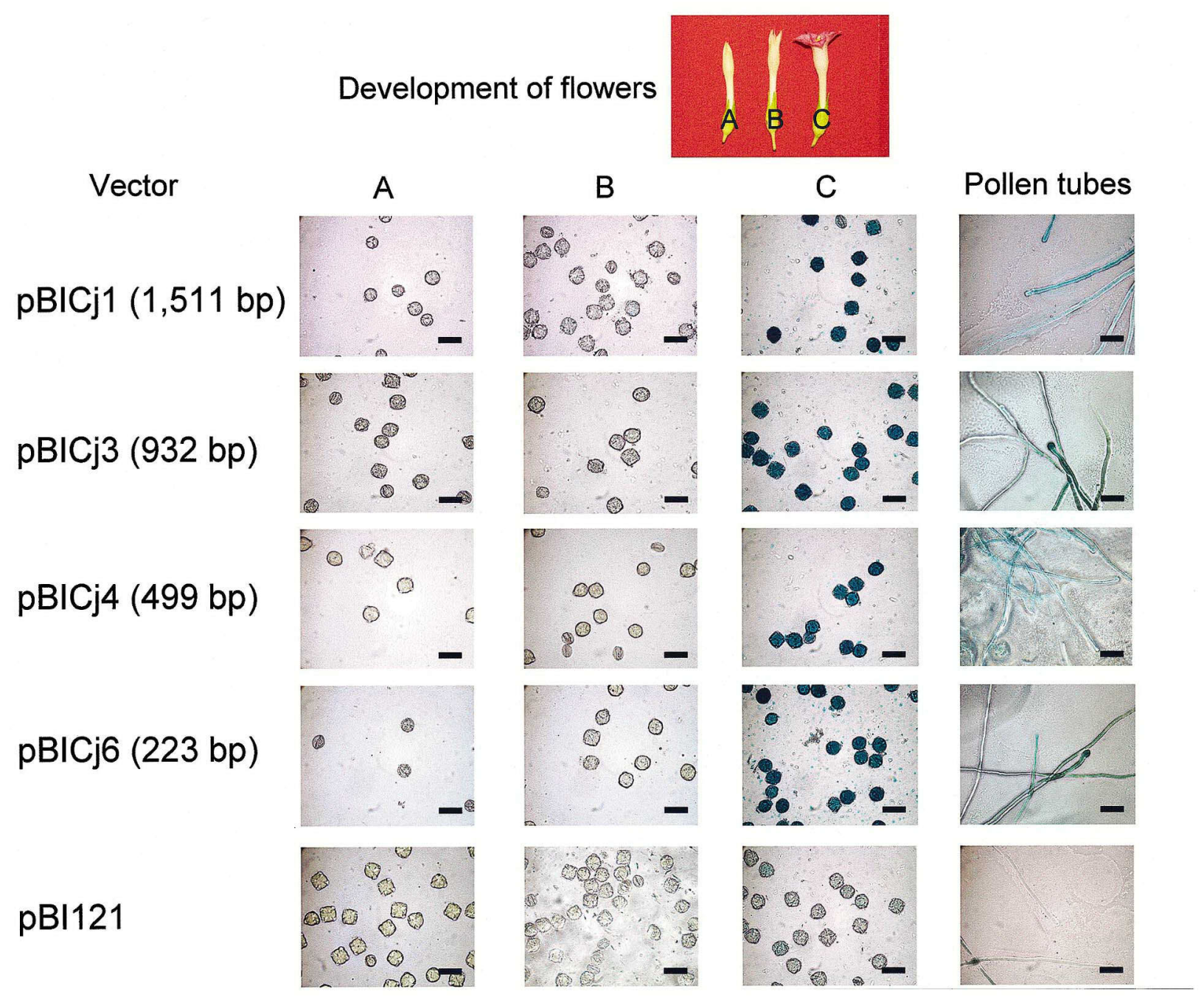

Fig. 3 Histochemical analyses of the expression of GUS in transgenic pollen grains and pollen tubes. Pollens and pollen tubes from transgenic tobacco plants that harbored four different Cry $j 1$ promoter-GUS constructs (pBICj1, pBICj3, pBICj4, and pBICj6) and a positive-control construct (pBI121) were stained with X-Gluc. Pollen at different stages of maturation was prepared from flowers at three different stages of maturation (A, B, and C). Pollen tubes were stained after the germination of mature pollen in vitro. Bars $=50 \mu \mathrm{m}$. 
is $\mathrm{A}$ or $\mathrm{C}$ ) found in the promoter sequences of angiosperm genes that express preferentially in tobacco and tomato pollens (Twell et al., 1991; Rogers et al., 1992; Kulikauskas and McCormick, 1997; Rogers et al., 2001). Eleven copies of the core sequences (GAAA) in the GAAA motif (AGAAAT$A A T)$ for pollen-specific expression found in the lat52 gene, which functions at a late stage in the development of tomato pollen, were also found in the promoter sequence of Cry $j$ 1. Furthermore, the elements CCACCACAACAC and CCACCAAAACGT, from positions -131 to -120 and -398 to 387 , respectively, were identical to the consensus sequence and identical at 11 out of 12 positions to the consensus target sequence (CCACMANMNYMN, where $M$ is $A$ or $C ; N$ is $A, C, G$, or $T$; and $\mathrm{Y}$ is $\mathrm{C}$ or $\mathrm{T}$ ) of a LIM-like protein (Ntlim1) of tobacco, respectively. This protein was identified as a transcription factor for PAL box-binding protein (Kawaoka et al., 2000). The gene for a plant LIM protein, PLIM-1, is expressed specifically in the pollen of sunflower (Baltz et al., 1992).

To examine whether the isolated sequence could confer tissue - and/or organ-specific expression and whether the putative cis elements described above might control gene expression, we fused Cry $j l$ promoter sequences of different lengths to the gene for GUS and used the resultant constructs to transform tobacco plants. Three promoter fragments of distinct lengths (932 bp, $499 \mathrm{bp}$, and $223 \mathrm{bp}$ ) were prepared from the longest amplified fragment (1,511 bp; clone 52; accession number AB080241) by digestion with a restriction endonuclease or PCR, as described below. To obtain the 932-bp fragment, the longest fragment that cloned into pT7Blue vector was blunt-- end ligated. In addition, 499 and 223-bp fragments were amplified by PCR using the longest fragment as a template. Each amplified fragment was cloned into pT7blue and sequenced completely. To produce promoter-GUS constructs, these four different fragments were recovered from the cloning vectors by digestion with HindIII and Bam HI, and the cauliflower mosaic virus $35 \mathrm{~S}$ (CaMV35S) promoter in pBI121 was replaced by these fragments. The resultant constructs were designated pBICj1 (1,511 bp), pBICj3 (932 bp), pBICj4 (499 bp), and pBICj6 (223 bp), and used to transform tobacco plants by an Agrobacterium-mediated method (Fig. 1). Transgenic plants transformed with pBI121, which includes a GUS gene driven by the CaMV35S promoter, were also produced as positive controls.

To characterize the qualitative expression of the Cry $j 1$ promoter-GUS constructs, we examined GUS activity in several organs and tissues (petal, sepal, stamen, pollen, pollen tube, leaf, and stem) of transgenic tobacco plants using the method described by Twell et al. (1990). This was initially examined using a histochemical assay of GUS activity in flower, leaf, and stem of the transgenic tobacco transformed with pBICj1. Fig. $2 \mathrm{~A}$ provides evidence for specific GUS expression in whole anthers containing pollen. This specific expression in the anthers disappeared after dispersion of pollen grains (Fig. 2B). No activity could be detected in leaf (Fig. 2C) and stem (data not shown) of the transgenic plant, suggesting that GUS expression in the transgenic plant is restricted to pollens and tapetum. Although we could not confirm GUS expression in tapetum tissue of the present transgenic plant, our results demonstrated clearly that, in addition to the longest promoter sequence $(1,511$ bp), all the sequences tested were able preferentially to direct expression in pollen grains that had been released from anthers (Fig. 3). Histochemical staining of flowers at distinct stages of maturity also indicated that expression in pollen was dependent on the extent of maturation. Fig. 3 shows clearly that expression was higher in mature pollen (column C) than in immature pollen (columns $A$ and B). Furthermore, expression of GUS was detected in pollen tubes after germination in vitro (Fig. 3). Fukui et al. (2001) reported that Cry $j I$ were expressed in male strobili of $C$. japonica from a long time before pollen maturation. In addition, Miki-Hirosige et al. (1994) demonstrated that Cry j 1 protein was localized in the cell wall of pollen grain at an early stage of pollen development as well as in the cytosol of pollen and in outer parts of the pollen cell wall such as sexines and orbicles that are derived from the tapetum. Our present results and the data obtained in the previous studies described here suggest that Cry $j 1$ protein plays an important role in both pollen development and fertilization. It is possible that Cry $j 1$ protein is involved in cell wall modification during pollen development and in pollen tube elongation, since the protein exhibits pectin lyase activity (McCormick, 1991).

In addition to the above qualitative analysis, we used a fluorometric assay (Morikawa et al. 1999) to quantitatively analyze GUS activity in mature pollen grains of $T_{0}$ transgenic plants and in leaf and stem tissues of $T_{1}$ plants. As shown in Table 1 , the fluorometric GUS activity in pollen transformed with pBICj1 was significantly higher than that in pollen transformed with the other GUS-fusion constructs $(P<0.1$ for $\mathrm{pBICj} 3$, and $P<0.01$ for pBICj4, pBICj6, and pBI121). Likewise, pollen of plants transformed with $\mathrm{pBICj} 3$ had higher activity 
Table 1. GUS assay of the tissues transformed with different promoter - GUS constructs

\begin{tabular}{|c|c|c|c|}
\hline & \multicolumn{3}{|c|}{$\begin{array}{l}\text { GUS activity } \\
\left\{4-\text { MU pmole } \min ^{-1}(\text { mg protein })^{-1}\right\}^{1}\end{array}$} \\
\hline & Mature pollen & Leaf & Stem \\
\hline pB1121 & $80 \pm 43^{3}$ & $690 \pm 61^{7}$ & $835 \pm 41^{9 ?}$ \\
\hline pBlCj1 & $4493 \pm 2019^{4 !}$ & $51 \pm 6^{8)}$ & $52 \pm 8^{81}$ \\
\hline $\mathrm{pBlCj} 3$ & $1476 \pm 84^{5)}$ & ND & ND \\
\hline $\mathrm{pBlCj4}$ & $346 \pm 82^{6)}$ & ND & $\mathrm{ND}$ \\
\hline pBlCj6 & $340 \pm 8^{6}$ & $\mathrm{ND}$ & ND \\
\hline Wild type ${ }^{2)}$ & ND & $61 \pm 8^{81}$ & $51 \pm 1^{b}$ \\
\hline
\end{tabular}

${ }^{1)}$ The activity is expressed in terms of the amount of 4-methylumbelliferone (4-MU) produced, as $\mathrm{pmole} / \mathrm{min} / \mathrm{mg}$ protein (mean \pm standard error; $\mathrm{n}=3$ ).

2) Non-transgenic tobacco plant.

3)-9) Different superscripts indicate significant differences among results $(\mathrm{P}<0.1)$.

ND, not determined.

than did pollen from plants transformed with pBICj4, pBICj6, and pBI121 (P<0.01). These results indicate that there are at least two independent cis elements that contribute to pollen-associated expression in the Cry $j 1$ promoter; namely, sequences between positions $-1,511$ and -935 , and sequences between positions -932 and -499 . In contrast to high GUS activity in mature pollens, the activities in leaf and stem tissues of the plant with pBICj1 were as low as those in wild-type tobacco. These results are consistent with the data from the histochemical GUS assay.

There were no obvious qualitative or quantitative differences between the results obtained with the pBICj4 $(-499$ to -1$)$ and pBICj6 $(-223$ to -1$)$ constructs, indicating that the $56 / 59-$ like box $(-378$ to 371 ) might not be an active element in angiosperms. The results of histochemical and fluorometric assays indicated that cis-regulatory element(s) for temporal and spatial expression of the gene are located within 223 bp of the $5^{\prime}$ untranslated regions. In addition, it is likely that the 11 bp consensus sequence found in the untranslated sequences of both the Cry $j I$ and Cry $j 2$ genes, and the sequence that resembles a LIMbinding element $(-131$ to -120$)$ are candidate $c i s$ elements for pollen-associated expression in $C$. japonica. Even though our present data do not reveal the definitive cis elements required for the qualitative and quantitative control of expression in pollen, further studies of cis elements in the Cry $\mathrm{j} I$ and $C r y j 2$ promoters should provide more information on the activities of gymnosperm cis elements in angiosperms and about sequence differences between promoter elements of gymnosperm and those of angiosperms that play the same roles in pollen-associated expression. Although further analysis is required to confirm GUS expression in tapetum tissue of transgenic tobacco, we are now attempting to produce transgenic $C$. japonica with a construct for suppression of $C r y j 1$ expression using both the isolated promoter sequence and the sequences of Cry $j 1$ cDNA. The availability of Cry $j 1$-suppressed $C$. japonica should contribute to the prevention of Sugi pollinosis in Japan.

\section{Acknowledgements}

The authors thank Dr. Katsuaki Ishii (Forestry and Forest Products Research Institute, Tsukuba) for supplying $C$. japonica tissue and seeds. The authors gratefully acknowledge Yuichirou Ohtsuka, Fumiko Honaga, and Mikiko Uesugi for their technical assistance. This study was supported in part by a Grant-in-Aid for Research for the Future Program (JSPS-RFTF 96L00605) from the Japan Society for Promotion of Science.

\section{References}

Baltz, R., Domon, C, Pillay, D. T. N., Steinmetz, A., 1992. Characterization of a pollen-specific cDNA from sunflower encoding a zinc finger protein. Plant J., 2: $713^{-}$ 721.

Brown, S. M., Crouch, M. L., 1990. Characterization of a gene family abundantly expressed in Oenothera organensis pollen that shows sequence similarity to polygalacturonase. Plant Ce11, 2: 263-274.

Budelier, K. A., Smith, A. G., Gasser, C. S., 1990. Regulation of a stylar transmitting tissue- specific gene in wild-type and transgenic tomato and tobacco. Mol. Gen. Genet., 224: 183-192.

Fukui, M., Futamura, N., Mukai, Y., Wang, Y., Nagao, A., Shinohara, K., 2001. Ancestral MADS box genes in Sugi, Cryptomeria japonica D. Don (Taxodiaceae), homologous to the B function genes in angiosperms. Plant Cell Physiol., 42: $566^{\sim} 575$.

Futamura, N., Mukai, Y, Sakaguchi, M., Yasueda, H., Inouye, S., Midoro-Horiuti, T, Goldblum, R. M., Shinohara, K., 2002. Isolation and characterization of CDNAs that encode homologs of a pathogenesis - related protein allergen from Cryptomeria japonica. Biosci. Biotechnol. Biochem., 66: 2495-2500.

Horiguchi, S., Saito, Y., 1964. Japanese cedar pollinosis in Nikko, Japan. Allergy, 13: 16-18.

Kawaoka, A., Kaothien, P., Yoshida, K., Endo, S., Yamada, K., Ebinuma, H., 2000. Functional analysis of tobacco LIM protein Ntlim1 involved in lignin biosynthesis. Plant J., 22: 289-301.

Kim, S.-R., Finkel, D., Chung, Y.-Y., An, G., 1994. Abundance patterns of lily pollen cDNAs: characterization of three pollen preferential cDNA clones. Sex. Plant Reprod., 7: 76-86. 
Kulikauskas, R., McCormick, S., 1997. Identification of the tobacco and Arabidopsis homologues of the pollenexpressed LAT59 gene of tomato. Plant Mol. Biol., 34: $809-814$

McCormick, S., 1991. Molecular analysis of male gametogenesis in plants. Trends Genet., 7: 298-303.

Miki-Hirosige, H., Nakamura, S., Yasueda, H., Shida, T., Takahashi, Y., 1994. Immunocytochemical localization of the allergenic proteins in the pollen of Crypiomeria japonica. Sex. Plant Reprod., 7: 95-100.

Morikawa, M., Yamakawa, T., Washino, T., Kodama, T., Igarashi, Y., 1999. Delayed recovery of $\beta$-glucronidase activity driven by an Arabidopsis heat shock promoter in heat-stressed transgenic Nicotiana plumbaginifolia. Plant Cell Rep., 19: 473- 497.

Mu, J. H., Stains, J. P., Kao, T., 1994. Characterization of a pollen-expressed gene encoding a putative pectin esterase of Petunia inflata. Plant Mol, Biol,, 25: $539-$ 544.

Niogret, M. F., Dubald, M., Mandaron, P., Mache, R., 1991. Characterization of pollen polygaracturonase encoded by several cDNA clones in maize. Plant Mol. Biol., 17: $1155-1164$.

Rafner, T., Griffith, I. J., Kou, M.-C., Bonds, J. F, Rogers, B. L., Klapper, D. G., 1991. Cloning of Amba I (Antigen E), the major allergen family of short ragweed pollen. J. Biol. Chem., 226: $1229-1236$.

Rogers, H. J., Harvey, A., Lonsdale, D. M., 1992. Isolation and characterization of a tobacco gene with homology to pectate lyase which is specifically expressed during microsporogenesis. Plant Mol. Biol., 20: 493-502.

Rogers, H. J., Bate, N., Combe, J, Sullivan, J., Sweetman, J., Swan, C., Lonsdale, D. M., Twell, D., 2001. Functional analysis of cis-regulatory elements within the promoter of the tobacco late pollen gene $\mathrm{g} 70$. Plant Mol. Biol., 45: $577-585$.

Sakaguchi, M., Inouye, S., Taniai, M., Ando, S, Usui, M., Matuhasi, T., 1990. Identification of the second major allergen of Japanese cedar pollen. Allergy, 45: $309-$ 312 .

Shibamura, K., Nishimura, Y., 1991. Japan cedar pollinosis on the immunogenetics. Kagaku, 61:93-97.

Sone, T., Komiyama, N., Shimizu, K., Kusakabe, T.,
Morikubo, K., Kino, K., 1994. Cloning and sequencing of CDNA coding for Cry $j I$, a major allergen of Japanese cedar pollen. Biochem. Biophys. Res. Commun., 199: 619-625.

Taniai, M., Ando, S., Usui, M., Kurimoto, M., Sakaguchi, M., Inouye, S., Matuhasi, T., 1988. N-terminal amino acid sequence of a major allergen of Japanese cedar pollen (Cry j I). FEBS Lett, 239: 329-332.

Taniguchi, Y., Ono, A., Sawatani, M., Nanba, M., Kohno, K., Usui, M., Kurimoto, M., Matuhasi, T., 1995. Cry j I, a major allergen of Japanese cedar pollen, has pectate Jyase enzyme activity. Allergy, 50: 90-93.

Turcich, M. P., Hamilton, D. A., Mascarenas, J. P., 1993. Isolation and characterization of pollen-specific maize genes with sequence homology to ragweed allergens and pectate lyases. Plant Mol. Biol, 23: $1061-1065$.

Twell, D., Yamaguchi, J., McCormick, S., 1990. Pollen specific gene expression in transgenic plants: coordinate regulation of two different tomato gene promoters during microsporogenesis. Development, 109: 709 713.

Twell, D., Yamaguchi, J., Wing, R. A., Ushiba, J., McCormick, S., 1991. Promoter analysis of genes that are coordinately expressed during pollen development reveals pollen-specific enhancer sequences and shared regulatory elements. Genes Dev., 5: 496-507.

Wakeley, P. R., Rogers, H. J., Rozycka, M, Greenland, A. J., Hussey, P. J., 1998.' A maize pectin methyltransferase-like gene, $Z m C 5$, specifically expressed in pollen. Plant Mol. Biol., 37: 187-192,

Wang, Y., Mukai, Y., Fukui, M., Futamura, N., Nagao, A., Shinohara, K., 1998. Pollen-specific expression of the gene for an allergen, Cry $\mathrm{j} 1$, in Cryptomeria japonica. J. For. Res., 3: 131-134.

Wing, R. A., Yamaguchi, J., Larabel1, S. K., Ursin, V. M., McCormick, S., 1989. Molecular and genetic characterization of two pollen-expressed genes that have sequence similarity to pectate lyases of the plant pathogen Erwinia. Plant Mol. Biol., 14: 17-28.

Yasueda, H., Yui, Y., Shimizu, T., Shida, T., 1983. Isolation and partial characterization of the major allergen from Japanese cedar (Cryptomeria japonica) pollen. J. Allergy Clin. Immunol., 71: 77-86. 